\title{
Study of non-linear heat transfer problems
}

\author{
A. Jordan, S. Khaldi, M. Benmouna and A. Borucki \\ Institut National de l'Enseignement Supérieur, Département de Physique, B.P. 119, Tlemcen, Algeria
}

(Reçu le 6 mars 1986, révisé le 30 septembre, accepté le 2 octobre 1986)

\begin{abstract}
Résumé. - L'équation non linéaire de transfert de chaleur dans un conducteur électrique de forme cylindrique est résolue à la fois numériquement et analytiquement à l'aide de la méthode de linéarisation optimale. Nous avons considéré le cas général où la conductivité thermique, la chaleur spécifique et la résistivité électrique sont des fonctions linéaires de la température. Une méthode de linéarisation optimale portant sur deux paramètres est présentée.
\end{abstract}

\begin{abstract}
The non linear heat transfert equation for an electrical cylindrical conductor is solved both numerically and analytically using the optimal linearization method. We have considered the general case where the thermal conductivity, the specific heat and the electrical resistivity are linear functions of temperature. A two parameters linearization method is presented.
\end{abstract}

\section{Introduction.}

Applications of non linear heat transfer problems can be found in various physical systems such as electrical conductors, solar systems, heat exchangers, etc... It is therefore very important to know the temperature distribution in these systems in order to obtain a good efficiency and avoid eventual malfunctions of electrical apparatus due to heat dissipation. We have recently examined some of these problems considering essentially cylindrical electrical conductors $[1,2]$, for which the most general equation governing the time and spatial distribution of the temperature $T$ is :

$$
\begin{aligned}
& \lambda(T)\left[\frac{\partial^{2} T}{\partial r^{2}}+\frac{1}{r} \frac{\partial T}{\partial r}\right]+\frac{\mathrm{d} \lambda(T)}{\mathrm{d} T}\left(\frac{\partial T}{\partial r}\right)^{2}- \\
&-\delta_{0} C(T) \frac{\partial T}{\partial t}=-\rho(T) j^{2},
\end{aligned}
$$

where $\lambda(T), C(T), \rho(T)$ and $\delta_{0}$ are the thermal conductivity, the specific heat, the electrical resistivity, and the mass density of the conductor, respectively. These parameters are, in the general case, all functions of temperature. $J$ is the current density assumed to be constant. We have already solved this problem assuming $[2,3]$ :

$$
\begin{aligned}
& \rho(T)=\rho_{0}(1+\alpha T), \\
& \lambda(T)=\lambda_{0}\left(1+\alpha^{\prime} T\right),
\end{aligned}
$$

and $C(T)=C_{0}$. The boundary and intial conditions which are usually used to solve this problem are :

$$
\begin{gathered}
\left.\frac{\partial T}{\partial r}\right|_{r=0}=0, \\
\left.\lambda(T) \frac{\partial T}{\partial r}\right|_{r=r_{0}}=-\varepsilon T\left(r_{0}\right), \\
T(r, t=0)=0, \quad 0 \leqslant r \leqslant r_{0},
\end{gathered}
$$

where $r_{0}$ is the radius of the conductor and $\varepsilon$ the convective heat transfer coefficient. In this case equation (1) becomes a non linear partial differential equation which we have solved both numerically and by using an optimal linearization method. For more details of this work, the reader is referred to references [1,2]. It appears by looking at $C(T)$ for standard electrical conductors, that in the region of temperature of interest to us, we must take the variation of the specific heat with temperature into account. This is the purpose of the present work where we let :

$$
C(T)=C_{0}(1+\beta T) .
$$

In the forthcoming section, we present the general formalism for solving such a problem which is based on :

(i) An exact numerical resolution using Runge-Kutta method.

(ii) A two parameter optimal linearization method. 
2. Resolution of the non linear heat transfer equation.

2.1 EXACT NUMERICAL RESOLUTION. - The numerical method which we use in this work is similar to the one applied in references $[1,2]$. The only difference is that we take into account the variation of $\lambda, \rho$ and $C$ with the temperature simultaneously. We use the
Runge-Kutta method to solve the space distribution of temperature, and the finite difference method to obtain its time evolution. We also choose the same physical parameters as the ones given by equations (22) and (24) of reference [2]. It is perhaps usefull to recall the equation to be solved numerically and which can be deduced immediately from equation (1) and put in the following form :

$$
\frac{\mathrm{d}^{2} T_{i+1}}{\mathrm{~d} r^{2}}=-\frac{1}{r} \frac{\mathrm{d} T_{i+1}}{\mathrm{~d} r}-\frac{\alpha^{\prime}}{1+\alpha^{\prime} T_{i+1}}\left(\frac{\mathrm{d} T_{i+1}}{\mathrm{~d} r}\right)^{2}+\frac{C_{0} \delta_{0}}{\lambda_{0}} \frac{1+\beta T_{i+1}}{1+\alpha^{\prime} T_{i+1}}\left(\frac{T_{i+1}-T_{i}}{\tau}\right)-\frac{j^{2} \rho_{0}}{\lambda_{0}} \frac{1+\alpha T_{i+1}}{1+\alpha^{\prime} T_{i+1}}
$$

$i=0,1,2, \ldots, N$ and $T_{0}=0$ for $0 \leqslant r \leqslant r_{0}$.

The results are displayed in figure 1 which shows the variations of the maximum temperature as a function of time for $C(T)=C_{0}$, i.e., a constant specific heat (curve 1) and $C(T)=C_{0}(1+\beta T)$ where $\beta=6.68 \times 10^{-4}\left[\mathrm{~K}^{-1}\right]$ (curve 2). It shows that for such a small value of $\beta$ the effect of the variation of the heat capacity with temperature is rather small (less than $2.5 \%)$. However, if for some other conductors $\beta$ is larger, this effect should be more important. One also notes, as expected, that the variation of the heat capacity with temperature does not affect the steady state temperature distribution. This is easily understood if one looks at equation (1) where the term proportional to $\partial T / \partial t$ is set to be zero. We also recall that this numerical resolution is given for a number of Biot larger that 1 (i.e. $B i=1.277$ ) corresponding to a significant decrease of temperature within the conductor. This numerical resolution obtained here will be considered as the exact result of equation (1), in the following sections. We shall seek now an approximate method to solve analytically equation (1) which is

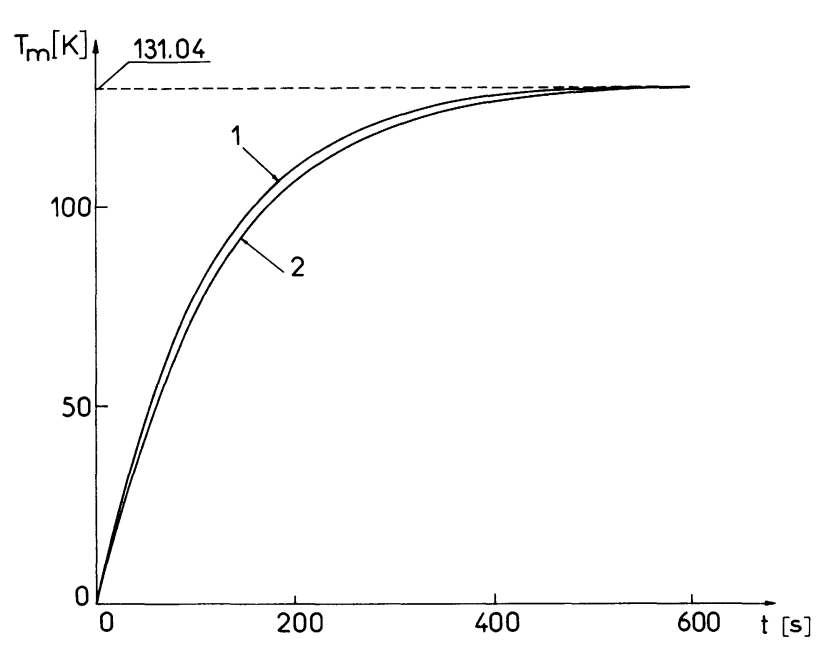

Fig. 1. - The variation of $T_{\mathrm{m}}[\mathrm{K}]$ with time $t[\mathrm{~s}]$ for $\mathrm{Al}$ in the case where $B i=1.277, \lambda(T)=\lambda_{0}\left(1+\alpha^{\prime} T\right)$, $\rho(T)=\rho_{0}(1+\alpha T)$ and: Curve (1): $C(T)=C_{0}$; Curve (2) : $C(T)=C_{0}(1+\beta T)$. This figure displays the exact numerical resolution for both curves. based on the optimal linearization theory. The predictions of the latter method will be compared with the exact numerical resolution.

\subsection{A TWO PARAMETER OPTIMAL LINEARIZATION} METHOD. - This method consists of approximating the non linear heat transfer equation (1) by a linear optimal equation which can be solved analytically. The basic idea of this method has already been explained in several instances. However, in the present case the problem is slightly different in the sense that we need to optimize a functional which depends on two parameters as opposed to previous cases where we had only one parameter. The functional in the present case is written as :

$$
\mathfrak{J}\left(K_{1}, K_{2}\right)=\int_{0}^{\infty} \int_{0}^{r_{0}} \Delta^{2} \mathrm{~d} r \mathrm{~d} t
$$

where $\Delta$ is defined by :

$$
\begin{aligned}
& \Delta=\left[K_{1}-\lambda(T)\right]\left[\frac{\partial^{2} T}{\partial r^{2}}+\frac{1}{r} \frac{\partial T}{\partial r}\right]- \\
& -\frac{\mathrm{d} \lambda(T)}{\mathrm{d} T}\left(\frac{\partial T}{\partial r}\right)^{2}-\left[K_{2}-\delta_{0} C(T)\right] \frac{\partial T}{\partial t}
\end{aligned}
$$

We note here that the upper bound of the time integration in the functional is taken to infinity as opposed to an earlier definition $[1,2]$ where we have introduced an arbitrary time $t_{1}$. This was simply defined as the time at which the steady state is reached within a certain accuracy. Substituting expression (10) into equation (9) yields.

$$
\begin{aligned}
\mathfrak{J}\left(K_{1}, K_{2}\right) & =A K_{1}^{2}-2 K_{1}[B+C-E]+ \\
+ & H K_{2}^{2}+2 K_{2}[F+G-M]-2 K_{1} K_{2} N+ \\
& + \text { constant terms , }
\end{aligned}
$$

where the coefficients $A, N, \ldots$ are expressed as integrals involving $T, \lambda(T), C(T)$ and their derivatives. We do not write them explicitely because they are lengthy and they can be derived fairly easily. We must note however that their calculation requires 
the knowledge of an expression of $T$ as a function of $r$, and $t$. Following our usual procedure described earlier $[1,2]$, we use the following approximate form which is deduced from the solution of the linear equation :

$$
T_{\mathrm{L}}(r, t)=a_{1}\left(1-\mathrm{e}^{-b_{1} t}\right) J_{0}\left(\frac{\xi_{1}}{r_{0}} r\right),
$$

where $a_{1}, b_{1}$ and $\xi_{1}$ can be found in earlier papers by Hoffer and us $[2,4]$. The problem is to find the parameters $K_{1}^{*}, K_{2}^{*}$ that optimize this functional. That is, one seeks the solution of the following sets of equations :

$$
\begin{aligned}
& \frac{\partial J}{\partial K_{1}}=0=A K_{1}^{*}-N K_{2}^{*}-(B+C-E), \\
& \frac{\partial J}{\partial K_{2}}=0=-N K_{1}^{*}+H K_{2}^{*}+(F+G-M) .
\end{aligned}
$$

Solving this set in terms of $K_{1}^{*}, K_{2}^{*}$ gives :

$$
\begin{aligned}
& K_{1}^{*}=\lambda_{0}\left[1+\alpha^{\prime} E_{1}\right], \\
& K_{2}^{*}=\gamma_{0}\left[1+\beta F_{1}\right]+\alpha^{\prime} E_{2},
\end{aligned}
$$

where $\gamma_{0}=\delta_{0} C_{0}$ and $E_{1}, E_{2}, F_{1}$ are given by :

$$
\begin{aligned}
& E_{1}=a_{1} \frac{\int_{0}^{r_{0}} J_{0}^{3}\left(\frac{\xi_{1}}{r_{0}} r\right) \mathrm{d} r-\int_{0}^{r_{0}} \mathfrak{J}_{1}^{2}\left(\frac{\xi_{1}}{r_{0}} r\right) J_{0}\left(\frac{\xi_{1}}{r_{0}} r\right) \mathrm{d} r}{\int_{0}^{r_{0}} J_{0}^{2}\left(\frac{\xi_{1}}{r_{0}} r\right) \mathrm{d} r} \\
& E_{2}=\frac{\lambda_{0} a_{1} \xi_{1}^{2}}{3 b_{1} r_{0}^{2}} \frac{\int_{0}^{r_{0}} \mathfrak{J}_{1}^{2}\left(\frac{\xi_{1}}{r_{0}} r\right) J_{0}\left(\frac{\xi_{1}}{r_{0}} r\right) \mathrm{d} r-\int_{0}^{r_{0}} \mathfrak{J}_{0}^{3}\left(\frac{\xi_{1}}{r_{0}} r\right) \mathrm{d} r}{\int_{0}^{r_{0}} \mathfrak{J}_{0}^{2}\left(\frac{\xi_{1}}{r_{0}} r\right) \mathrm{d} r} \\
& F_{1}=\frac{a_{1}}{3} \frac{\int_{0}^{r_{0}} \mathfrak{J}_{0}^{3}\left(\frac{\xi_{1}}{r_{0}} r\right) \mathrm{d} r}{\int_{0}^{r_{0}} \mathfrak{J}_{0}^{2}\left(\frac{\xi_{1}}{r_{0}} r\right) \mathrm{d} r} .
\end{aligned}
$$

Therefore, the exact non linear equation (1) is approximated by the following linear equation :

$$
K_{1}^{*}\left[\frac{\partial^{2} T}{\partial r^{2}}+\frac{1}{r} \frac{\partial T}{\partial r}\right]-K_{2}^{*} \frac{\partial T}{\partial t}=-\rho_{0}(1+\alpha T) j^{2},
$$

and initial conditions (4), (5) and (6) in which $\lambda(T)$ is replaced by $K_{1}^{*}$. The solution can be written in the form :

$$
T(r, t)=\sum_{n=1}^{\infty} T_{n}(r)\left[1-\mathrm{e}^{-b_{n} t}\right],
$$

which can be solved analytically using the boundary

where :

$$
\begin{gathered}
T_{n}(r)=\frac{-\left[2 \varepsilon a \lambda_{0} / K_{2}^{*}\right] J_{0}\left(\frac{\xi_{n}}{r_{0}} r\right)}{b_{n}\left\{J_{0}\left(\xi_{n}\right)\left[\varepsilon-\xi_{n}^{2} \frac{K_{1}^{*}}{r_{0}}\right]-J_{1}\left(\xi_{n}\right)\left[\varepsilon+\frac{K_{1}^{*}}{r_{0}}\right] \xi_{n}\right\}} \\
b_{n}=\left[\frac{\xi_{n}^{2}}{r_{0}^{2}}-\frac{a \alpha \lambda_{0}}{K_{1}^{*}}\right] \frac{K_{1}^{*}}{K_{2}^{*}}, \quad a=\frac{j^{2} \rho_{0}}{\lambda_{0}}
\end{gathered}
$$

$\xi_{n}$ are the roots of the following equation :

$$
\mathfrak{J}_{0}(\xi)-\frac{K_{1}^{*}}{\varepsilon r_{0}} \xi J_{1}(\xi)=0
$$

where $J_{0}(\xi)$ and $\mathfrak{J}_{1}(\xi)$ are Bessel functions of the first kind. The comparison of this approximate solution and the exact numerical solution is given in figure 2 which displays the curves corresponding to cases using the same physical parameters defined earlier, in particular $\beta=6.68 \times 10^{-4}\left[\mathrm{~K}^{-1}\right]$ and $B i=1.277$. One ob- 


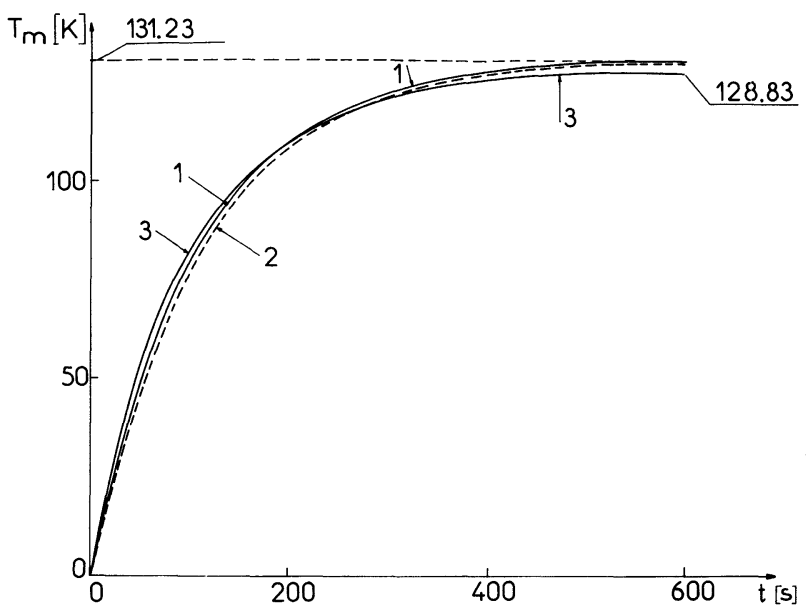

Fig. 2. - The variation of $T_{\mathrm{m}}[\mathrm{K}]$ with time $t[\mathrm{~s}]$ for $\mathrm{Al}$, in the case where $B i=1.277$ and: Curve (1): solution of the optimal linear equation (20), Curve (2): exact numerical solution of equation (1), Curve (3) : exact solution of the linear equation corresponding to $\lambda(T)=\lambda_{0} C(T)=C_{0}$ and $\rho(T)=\rho_{0}(1+\alpha T)$.

serves that the two curves are close to each other which implies that the optimal linearization method provides a good approximation for the non linear heat transfer equation. We have added in the same figure the case where the thermal conductivity and the specific heat are constants (curve 3 ). Although in all cases we have a relatively small departure from the exact solution corresponding to the general situation where $\lambda(T)$, $C(T)$ and $\rho(T)$ are functions of temperature, we see that the optimal linearization method is a good approximattion and provides a powerfull tool for solving analytically non linear partial differential equations.

If one wants to study the rate of time increase of the transient temperature, one considers the fundamental mode which corresporids to the first term of the sum in equation (21), i.e.,

$$
T(r, t)=T_{1}(r)\left[1-\mathrm{e}^{-t / \tau_{1}}\right],
$$

where the fundamental time constant $\tau_{1}$ is given by :

$$
\tau_{1}=\frac{K_{2}^{*} / a \lambda_{0}}{K_{1}^{*} \xi_{1}^{2} / r_{0}^{2} a \lambda_{0}-\alpha}
$$

Using the same physical parameters as in previous papers $[1,2]$, namely :

$$
\begin{aligned}
\alpha & =43 \times 10^{-4}\left[\mathrm{~K}^{-1}\right], \\
\alpha^{\prime} & =-3.4 \times 10^{-4}\left[\mathrm{~K}^{-1}\right], \\
\beta & =6.68 \times 10^{-4}\left[\mathrm{~K}^{-1}\right], \mathrm{Al} \text { at } 293[\mathrm{~K}] .
\end{aligned}
$$

One can calculate the quantities appearing in equation (26) and obtain :

$$
\begin{aligned}
K_{1}^{*} & =173,718\left[\mathrm{~W} \mathrm{~m}^{-1} \mathrm{~K}^{-1}\right], \\
K_{2}^{*} & =2507584,1\left[\mathrm{~J} \mathrm{~m}^{-3} \mathrm{~K}^{-1}\right], \\
a & =13.725 \times 10^{3}\left[\mathrm{~K} \mathrm{~m}^{-2}\right], \\
\xi_{1} & =1.394 \text { for equation }(24), \\
\xi_{1} & =1.375 \text { for the linear case }\left(\alpha^{\prime}=0, \beta=0\right) .
\end{aligned}
$$

This gives the following estimates for $\tau_{1}$ in various cases displayed in figure 2

\section{$108.30 \mathrm{~s}$ for the optimal curve 1}

$102.58 \mathrm{~s}$ for the linear curve 3 .

If one lets $\rho(T)=\rho_{0}$ constant, one finds that $\tau_{1}$ is drastically decreased to the value $\tau_{1}=70.56 \mathrm{~s}$.

It is interesting to note that the response of the system is faster in the case where the electrical resistivity is constant with temperature. This can be easily seen from equation (26), which shows the important effect of $\alpha$ on the value of the time constant $\tau_{1}$ for electrical conductors such as the one considered in this example.

\section{Conclusions.}

The aim of this paper was to generalize the optimal linearization method to the case where the main physical parameters of the system are functions of temperature. This implies the minimization of a functional with two parameters simultaneously. In earlier work, we have used a method to optimize one parameter only.

We have shown that this method provides an analytical solution wich approximates the exact one fairly well. Furthermore, we have defined a functional with respect to time and space integrations in which the upper bound of the time integration was infinity. This enabled us to avoid the introduction of an arbitrary time $t_{1}$ as done in an earlier paper [2]. This observation can also be made for the calculation of the constants $E_{1}, E_{2}$ and $F_{1}$. One can check that by taking $t_{1}$ to infinity in equation (16b) of reference [2] one obtains our result in equation (19). This also holds for equation (9) of reference [2] and $E_{1}$ in equation (17). 


\section{References}

[1] Berbar, B., Jordan, A., Benmouna, M., Steady state temperature distribution in a cylindrical electrical conductor: non-linear effects, Revue Phys. Appl. 18 (1983) 677-681.

[2] Jordan, A., Benmouna, M., Borucki, A., BouAyed, F., Transient State Temperature distribution in an cylindrical electrical conductor non-linear effects, Revue Phys. Appl. 21 (1986) 263-267.
[3] Laurent, M., Etude Comparative et critique de quelques méthodes de détermination des caractéristiques thermophysiques des solides conducteurs de la chaleur, Revue Gén. Therm. 238 (1981) 701-718.

[4] HoffER, O., Instationäre Temperaturverteilung in Einem Runddraht, Archiv Elektrotech 60 (1978) 319-325. 